\title{
Genetic interaction of the SMC complex with topoisomerase IV in Bacillus subtilis
}

\author{
Correspondence \\ Peter L. Graumann \\ pg32@biologie.uni-freiburg.de
}

Received 27 May 2005

Revised 3 August 2005

Accepted 15 August 2005

\author{
Serkalem Tadesse, ${ }^{1} \dagger$ Judita Mascarenhas, ${ }^{1}$ Bernd Kösters, ${ }^{2}$ \\ Andrej Hasilik ${ }^{2}$ and Peter L. Graumann ${ }^{1} \dagger$
}

Biochemie, Fachbereich Chemie, Hans-Meerwein-Straße ${ }^{1}$ and Institut für Physiologische Chemie, Karl-von-Frisch-Straße 12, Philipps-Universität Marburg, 35032 Marburg, Germany

\begin{abstract}
The role of topoisomerase IV (Topo IV) and of the structural maintenance of chromosomes (SMC) complex in chromosome compaction and in global protein synthesis was investigated. Lowering of the levels of Topo IV led to chromosome decondensation, while overproduction induced chromosome hyper-compaction, showing that Topo IV has an influence on the compaction of the whole chromosome, in a manner similar to that of the SMC protein, though different in mechanism. Increased synthesis of Topo IV in smc-deleted cells partially rescued the growth and condensation defect of the deletion, but not the segregation defect, revealing that the two systems interact at a genetic level. Two-dimensional gel investigations showed that global protein synthesis is highly aberrant in smc-deleted cells, and, to a different extent, also in cells lacking ScpA or ScpB, which form the SMC complex together with SMC protein. Overproduction of Topo IV partially rescued the defect in protein synthesis in smc mutant cells, indicating that Topo IV can restore the loss of negative supercoiling caused by the absence of SMC protein, but does not fully rescue the segregation defect. The data also show that the SMC protein has a dual function, in chromosome supercoiling and in active segregation.
\end{abstract}

\section{INTRODUCTION}

Chromosomes must be compacted to fit into the nucleus of the bacterial cell, and DNA topology must be regulated to allow transcription and replication to take place. DNA compaction is regulated by histones or histone-like proteins, which wind DNA around themselves, by topoisomerases, which affect supercoiling directly through changes in the linking number (the number of times DNA strands pass around each other), and by structural maintenance of chromosomes (SMC) proteins. Homeostasis of global supercoiling of the chromosome (which is negatively supercoiled in mesophilic bacteria) is regulated by the antagonistic action of topoisomerase II (DNA gyrase), which actively introduces negative supercoils, and of topoisomerase I (Topo I), which relaxes negative supercoils (Espeli \& Marians, 2004). There are two additional topoisomerases, whose function in global supercoiling is less clear. Topoisomerase III (Topo III) is not essential (in contrast to the other three enzymes), and appears to be involved in DNA recombination (Zhu et al., 2001), while topoisomerase IV

†Present address: Institut für Mikrobiologie, Stefan Meier Str. 19, Albert-Ludwigs Universität Freiburg, 79104 Freiburg, Germany.

Abbreviations: 2D, two-dimensional; SMC, structural maintenance of chromosomes; Topo I, topoisomerase I; Topo III, topoisomerase III; Topo IV, topoisomerase IV.
(Topo IV) is required for the separation of interlinked sister chromosomes (Kato et al., 1990), which arise during replication and DNA recombination, and which would resist complete segregation of sister chromosomes. In vitro, Topo IV (which consists of two subunits, products of parE and parC) preferentially relaxes positive supercoils and has a high decatenation activity, but its detailed contribution to chromosomal processes in vivo is still unclear.

SMC proteins are key players in various aspects of chromosome dynamics in most organisms. Their functions include cohesion of sister chromosomes, which is essential for stable segregation of chromosomes during mitosis, DNA double-strand break repair, chromosome compaction and maintenance of chromosome structure (Hirano, 2002). SMC proteins invariably act within protein complexes containing an SMC protein homodimer (in prokaryotic cells) or a heterodimer, and, additionally, non-SMC proteins. In prokaryotes, an SMC protein homodimer forms a complex with ScpA, belonging to the kleisin protein family, and with ScpB, both of which are widely conserved in bacteria and archaea (Mascarenhas et al., 2002; Soppa et al., 2002). SMC proteins have an unusual structure: an SMC protein monomer is composed of an ATPase head domain at one end, a long coiled-coil region, and a hinge domain at the other end. SMC protein dimers are formed through a strong and specific dimerization of the hinge domains, and are thus 
symmetrical molecules with a central flexible hinge, two coiled-coiled arms, and two ATPase-cassette head domains. DNA binding is mediated by embracing with the coiled-coil arms and most likely through ATP-dependent dimerization of the head domains, leading to ring closure around the DNA (Gruber et al., 2003; Volkov et al., 2003). In vitro, condensin can introduce positive writhe into DNA, that is it bends the DNA into a right-handed superhelix (Kimura et al., 1999), through an as yet ill-defined mechanism. This tension can be relieved by Topo I, the net result being that negative supercoiling is introduced into DNA. Indeed, in vivo, condensin and the prokaryotic SMC complex appear to induce chromosome compaction through the introduction of negative supercoiling into DNA. This has been demonstrated because: (a) the reduction of Topo I activity is a suppressor of a $m u k B$ (smc in Escherichia coli) deletion (Sawitzke \& Austin, 2000); (b) smc mutant cells are hypersensitive to inhibitors of DNA gyrase (Lindow et al., 2002a). The bacterial SMC complex localizes in two discrete centres, one within each cell half (Lindow et al., 2002b; Mascarenhas et al., 2002), while DNA is replicated by a centrally located replication machinery (Lemon \& Grossman, 2000). Thus, DNA moves through the central replisome, and replicated DNA strands are moved towards opposite cell poles, by an as yet unknown mechanism. Newly replicated DNA is probably locally condensed within the SMC centres, which organize chromosome layout and facilitate segregation (Graumann, 2000).

In this work, we wished to elucidate the connection between Bacillus subtilis Topo IV and the SMC complex. We found that overproduction of Topo IV suppressed the chromosome condensation defect of an smc deletion, as well as a defect in global protein synthesis caused by the loss of the SMC complex, but not its segregation defect, revealing an intricate connection between SMC protein and Topo IV in vivo.

\section{METHODS}

Growth conditions. E. coli XL-1 Blue (Stratagene) or B. subtilis strains were grown in Luria-Bertani (LB) rich medium supplemented with $50 \mu \mathrm{g}$ ampicillin $\mathrm{ml}^{-1}$ or other antibiotics, where appropriate. For microscopic analysis, Bacillus strains were grown in $\mathrm{S7}_{50}$ defined medium (Jaacks et al., 1989) complemented with $1 \%$ Casamino acids. For induction of the xylose promoter, $0.5 \%$ xylose was added to the medium, and glucose was exchanged for fructose and xylose in $\mathrm{S}_{50}$ medium. For induction of Phyperspank, IPTG was added. Antibiotics were used at the following final concentrations: chloramphenicol $(\mathrm{Cm}), 5 \mu \mathrm{g} \mathrm{ml}^{-1}$; spectinomycin (spec), $100 \mu \mathrm{g} \mathrm{ml}^{-1}$; Mls (erythromycin, $1 \mu \mathrm{g} \mathrm{ml}^{-1}$; lincomycin, $25 \mu \mathrm{g} \mathrm{ml}^{-1}$ ); kanamycin (kan), $10 \mu \mathrm{g} \mathrm{ml}^{-1}$; tetracycline (tet), $10 \mu \mathrm{g} \mathrm{ml}^{-1}$.

Construction of plasmids and bacterial strains. To regulate expression of parE and $\operatorname{parC}$, we constructed a strain with both genes under the control of the IPTG-inducible promoter Phyperspank. A $500 \mathrm{bp}$ sequence containing the $5^{\prime}$ region of parE was PCR-amplified and cloned into pJQ43 [(Quisel et al., 2001) in which the Phyperspank region had been introduced from pDR111 (kind gift of D. Rudner, Harvard Medical School)] using SphI and NheI sites, to generate pJM45. This plasmid was integrated into PY79 by single-crossover recombination to generate the clone ST16 using Cm and different concentrations of IPTG $\left(1-10^{-3} \mathrm{mM}\right)$. Since $10^{-1} \mathrm{mM}$ IPTG supported wild-type Topo IV activity, this concentration was used for strain passage and for further genetic manipulation. ST20 was generated by transforming ST16 with chromosomal DNA from PGA388 using kan.

Image acquisition. Fluorescence microscopy was performed on an Olympus AX70 microscope. Cells were mounted on agarose gel pads containing $\mathrm{S}_{50}$ growth medium on object slides. Images were acquired with a digital charge-coupled device (CCD) camera; signal intensities and cell length were measured using the Metamorph 5.0 program (Universal Imaging Corp., USA). DNA was stained with 4',6-diamidino-2-phenylindole (DAPI, final concentration $0 \cdot 2 \mathrm{ng} \mathrm{ml}^{-1}$ ) and membranes were stained with the vital membrane stain FM4-64 (Molecular Probes, final concentration $1 \mathrm{nM}$ ).

Two-dimensional (2D) gel electrophoresis. The different strains were grown at $23^{\circ} \mathrm{C}$ in $500 \mathrm{ml} \mathrm{LB}$ broth to $\mathrm{OD}_{600} 0 \cdot 5$. Cells were collected by centrifugation, washed with ice-cold sterile water, resuspended in buffer $\left(10 \mathrm{mM}\right.$ Tris/ $\mathrm{HCl}, 1 \mathrm{mg} \mathrm{MgCl}_{2} \mathrm{ml}^{-1}, 100 \mu \mathrm{g}$ DNase $\mathrm{ml}^{-1}, 100 \mu \mathrm{g}$ RNase $\mathrm{ml}^{-1}, 1.5 \mathrm{mM}$ PMSF) and disrupted by sonication. The supernatant was freeze-dried in liquid nitrogen and lyophilized. The freeze-dried protein sample was precipitated overnight using acetone, and dissolved in $125 \mu$ rehydration buffer [ $9 \mathrm{M}$ urea, $4 \%(\mathrm{v} / \mathrm{v})$ CHAPS, $2 \%(\mathrm{v} / \mathrm{v})$ ampholytes $\mathrm{pH} 4-7,0 \cdot 5 \%(\mathrm{v} / \mathrm{v})$ ampholytes $\mathrm{pH} 3-10,10 \mathrm{mM}$ DTT]. 2D gel eletrophoresis was performed using cylindrical tube gels $(3 \times 100 \mathrm{~mm})$, and $15 \%$ polyacrylamide slab gels for the second dimension, applying $50 \mu \mathrm{g}$ total protein from the cell extract, according to the protocol described in Klein et al. (2002). The gels were stained with silver nitrate and dried between two sheets of cellophane. The gels were scanned as 16 bit images (UMAX, PowerLook 2100XL scanner) and analysed with ProteomWeaver software version 2.1 (Definiens, Munich, Germany). The pattern of all of the gels was used to generate an average, and the spots detected were compared with one another in terms of their intensity. SPSS version 11.0 software was used for analysis of the raw data obtained from ProteomWeaver. An unpaired $t$ test was employed for testing the statistical significance of the different mutants on the $2 \mathrm{D}$ gels and to compare the mean protein spot intensities with one another.

\section{RESULTS}

\section{Levels of Topo IV influence global chromosome compaction}

Phyperspank is an IPTG-dependent promoter that allows for variation of transcriptional regulation over a wide range. To investigate if variation of the level of Topo IV has an influence on the final steps of chromosome segregation only, or on general DNA supercoiling, we placed the parE and $\operatorname{parC}$ genes (whose products form Topo IV) at their original position on the chromosome under the control of Phyperspank. Variation of the concentration of IPTG led to dramatic changes in cell morphology and chromosome structure. A concentration of $0.1 \mathrm{mM}$ IPTG allowed a normal growth rate during exponential phase (Fig. 1), and showed normal cell length and nucleoid morphology, compared to wild-type cells (Fig. 2C, compare with 2A). This concentration was therefore defined as the standard level of Topo IV, most closely resembling wild-type levels of expression. In Fig. 1, it is apparent that Phyperspank-driven transcription of the parC and parE genes led to a moderate 


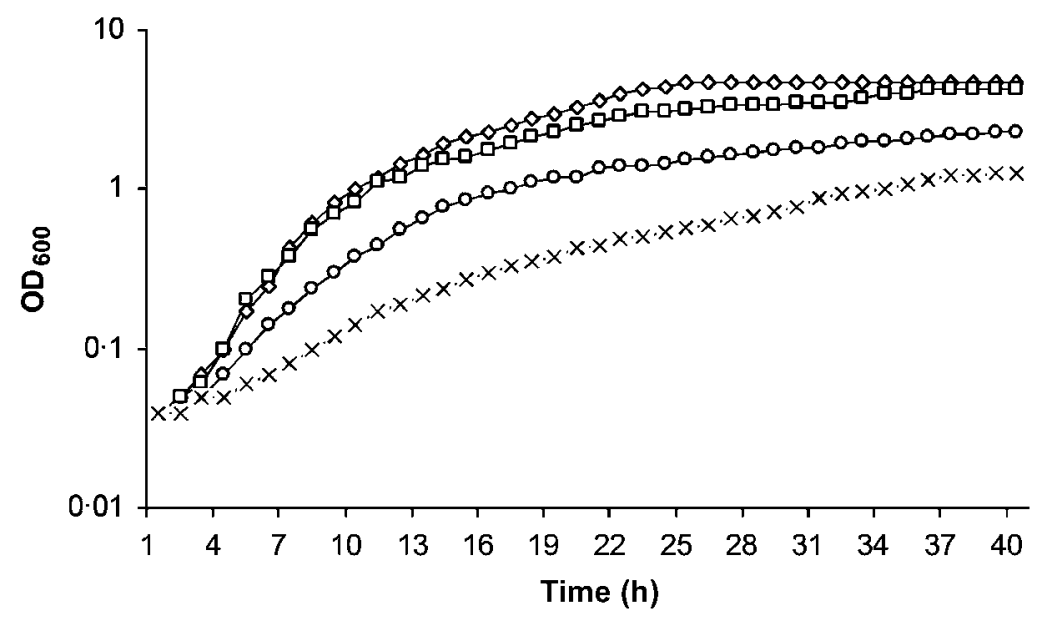

Fig. 1. Growth of wild-type and different mutant strains at $23^{\circ} \mathrm{C}$. $\diamond$, Wild-type cells; $\square$, wild-type cells in which parE and parC are under control of Phyperspank, growing at $0.1 \mathrm{mM}$ IPTG; $\bigcirc$, smc mutant cells in which parE and parC are under control of Phyperspank, growing at $1 \mathrm{mM}$ IPTG; $\mathrm{x}, \mathrm{smc}$ mutant cells.

growth defect as cells approached stationary phase, indicating that Topo IV levels might undergo a physiologically important change of expression at this growth phase in wildtype cells. Lower levels of IPTG induced more and more pronounced chromosome decondensation, accompanied by increasingly slower growth rate and larger cell size. At $0.01 \mathrm{mM}$ IPTG, chromosomes in $75 \%$ of the cells were considerably less condensed and hence occupied a larger space within the cells compared to wild-type cells (Fig. 2D), and at $0.001 \mathrm{mM} \mathrm{IPTG}$, chromosomes were spread through almost the entire cytosolic space in $>90 \%$ of the cells ( $>300$ cells analysed for each condition). Average cell size was $2 \cdot 5$-fold larger than that of wild-type cells, and two separate nucleoids were present in only $2 \%$ of the cells, as compared to $25 \%$ in wild-type cells, showing that lower levels of Topo IV lead to a severe compaction defect. Interestingly, however, even at the lowest concentration tested, only $1 \%$ anucleate cells were observed (Table 1 , $>300$ cells analysed for each condition), showing that even a low concentration of Topo IV can support enough chromosome decatenation to avoid complete missegregation before cell division. When cells were grown in the absence of inducer, anucleate cells arose at high frequency (Fig. 2F, Table 1), until cells eventually lysed, in agreement with earlier reports (Kato et al., 1990).

Conversely, growth of cells in $1 \mathrm{mM}$ IPTG (full induction of the Phyperspank promoter) induced pronounced nucleoid hypercondensation (Fig. 2B). In $45 \%$ of the cells, nucleoids occupied a considerably smaller cytosolic space, and in many cells had an almost-round morphology (indicated by arrowheads). Interestingly, chromosome hypercompaction led to only a moderate segregation defect: only $0.4 \%$ of the cells were anucleate (Table 1). Thus, varying the level of Topo IV strongly alters the degree of chromosome compaction in growing cells, suggesting that Topo IV influences global supercoiling, in addition to its function in decatenation at the final step of chromosome segregation (Espeli et al., 2003). This observation agrees well with inhibition studies in E. coli cells (Zechiedrich et al., 2000).

\section{An increase in synthesis of Topo IV partially suppresses an smc deletion}

The global effect of Topo IV on chromosome condensation led us to speculate that the defect in chromosome supercoiling due to an $s m c$ deletion could be suppressed by varying levels of Topo IV. To address this question, we moved the Phyperspank-parE construct into an smc null background and grew the cells at permissive temperature $\left(23^{\circ} \mathrm{C}\right)$ with different levels of IPTG. At $0 \cdot 1 \mathrm{mM}$ IPTG (which would correspond to an $s m c$ null background with a wild-type level of Topo IV), cells displayed a typical $s m c$ phenotype, that is, chromosomes were decondensed and anucleate cells arose at a frequency of 15-20\% (Fig. $2 \mathrm{H}$, indicated by arrowhead). Lowering the Topo IV levels in $s m c$ mutant cells further exacerbated the $s m c$ phenotype: nucleoids were further decondensed and extended, and $25 \%$ anucleate cells were counted at $0.01 \mathrm{mM}$ IPTG (Fig. 2I). Growth almost ceased at this level of inducer. In contrast, growth of mutant cells in the presence of $1 \mathrm{mM}$ IPTG resulted in markedly more condensed chromosomes in $80 \%$ of the cells (Fig. 2G). Chromosomes were still less condensed than in wild-type cells, but the difference was rather subtle. These experiments show that overproduction of Topo IV suppresses the chromosome-condensation defect in $s m c$ mutant cells. We also measured the growth of cultures from cells of the wild-type, $s m c$ mutant and $s m c$ mutant with overexpressed Topo IV. It is apparent from Fig. 1 that increased expression of Topo IV in an smc mutant background led to a higher growth rate than that of the $s m c$ deletion strain, but the rate of growth was still considerably lower than that of wild-type cells. Smc null cells overexpressing Topo IV were able to grow at $30^{\circ} \mathrm{C}$, but not at $37^{\circ} \mathrm{C}$, whereas $s m c$ null cells were only able to grow below $23^{\circ} \mathrm{C}$ (Table 1). Additionally, even with increased levels of Topo IV, 5-10\% anucleate cells were observed (Table 1). Thus, overproduction of Topo IV partially suppresses the loss of function of SMC protein, in that it almost fully rescues the condensation defect, but only partially reduces the severity of the segregation and growth defects. 
(A)

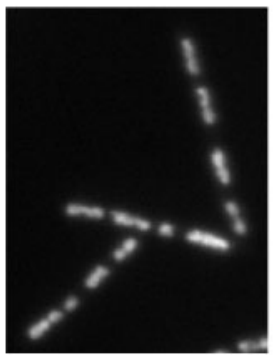

DNA

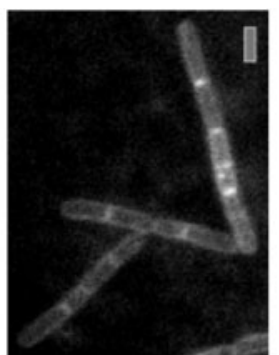

Membrane
(B)

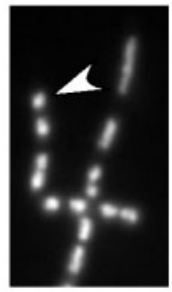

DNA

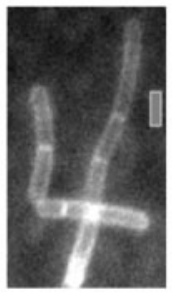

Membrane

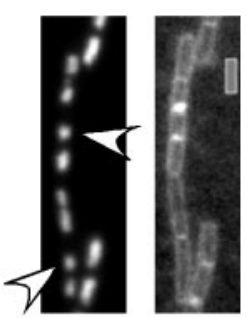

DNA Mem.
(C)

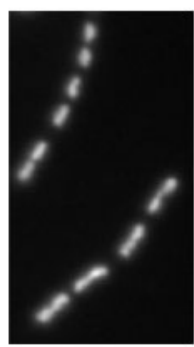

DNA

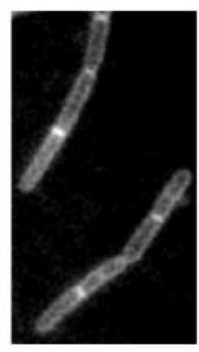

Membrane
(D)

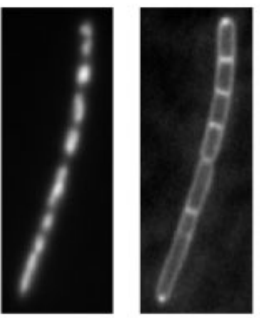

DNA Membrane
(E)

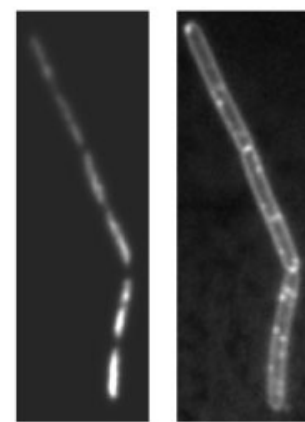

DNA Membrane

(F)

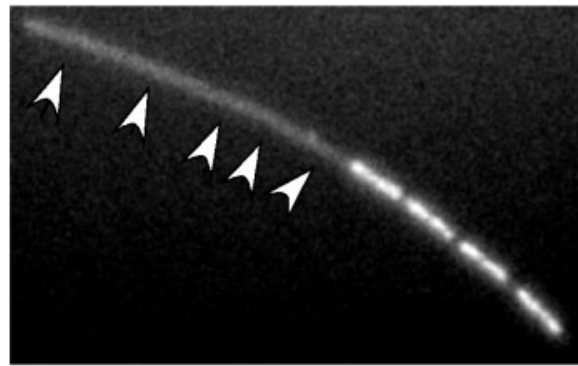

DNA

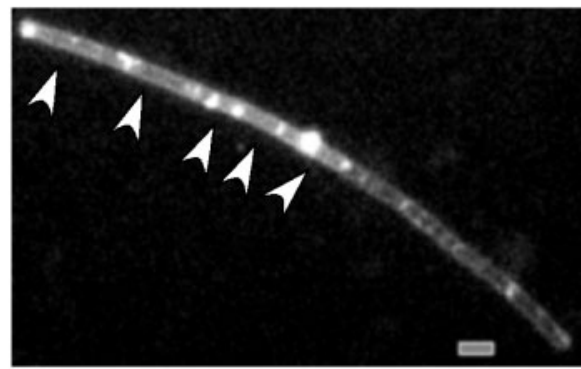

Membrane

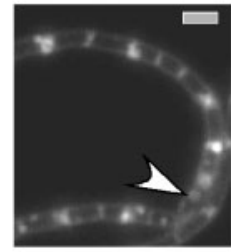

Membrane

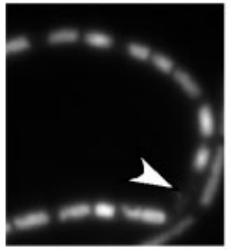

DNA
(H)

DNA
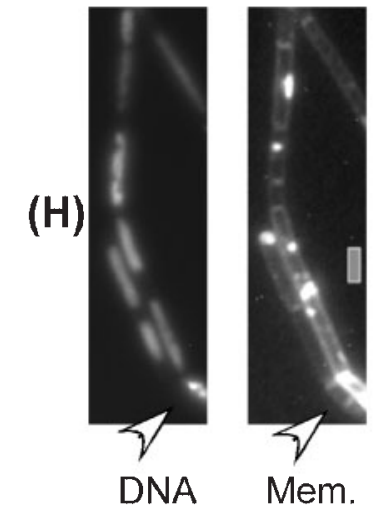

(I)
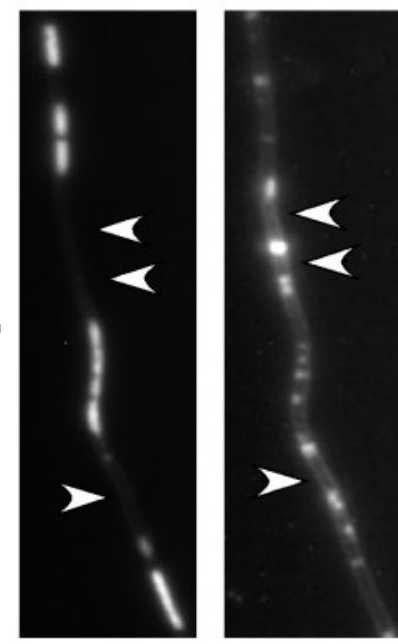

DNA

Membrane

Fig. 2. Effect of different levels of Topo IV on chromosome compaction, and suppression of an smc deletion. (A) Wild-type cells, (B-F) cells of ST16 (phyperspank-parE) growing at (B) $1 \mathrm{mM} \mathrm{IPTG,} \mathrm{(C)} 0.1 \mathrm{mM} \mathrm{IPTG,} \mathrm{(D)} 0.01 \mathrm{mM}$ IPTG, (E) $0.001 \mathrm{mM}$ IPTG or (F) no IPTG. (G-I) Cells of strain ST20 (phyperspank-parE, smc:: kan) growing at (G) $1 \mathrm{mM} \mathrm{IPTG,} \mathrm{(H)}$ $0.1 \mathrm{mM}$ IPTG or (I) $0.01 \mathrm{mM}$ IPTG. White arrowheads indicate anucleate cells; bar, $2 \mu \mathrm{m}$; Mem., membrane. 
Table 1. Growth and segregation defects in wild-type and mutant strains

\begin{tabular}{|c|c|c|c|c|c|}
\hline \multirow[t]{2}{*}{ Strain } & \multirow{2}{*}{$\begin{array}{l}\text { IPTG concentration } \\
\qquad(\mathrm{mM})\end{array}$} & \multirow{2}{*}{$\begin{array}{c}\text { Percentage } \\
\text { of anucleate cells } \\
\left(\text { at } 23^{\circ} \mathrm{C}\right)\end{array}$} & \multicolumn{3}{|c|}{ Growth at: } \\
\hline & & & $23^{\circ} \mathrm{C}$ & $30^{\circ} \mathrm{C}$ & $37^{\circ} \mathrm{C}$ \\
\hline PY79 (wild-type) & - & $<0 \cdot 001$ & + & + & + \\
\hline ST16 Phyper-parE & 1 & $0 \cdot 4$ & + & + & + \\
\hline ST16 Phyper-parE & $0 \cdot 1$ & $<0 \cdot 001$ & + & + & + \\
\hline ST16 Phyper-parE & $0 \cdot 001$ & 1 & + & + & + \\
\hline ST16 Phyper-parE & - & $35^{\star}$ & - & - & - \\
\hline $\mathrm{PG} \Delta 388(\Delta s m c)$ & - & $15 \cdot 5$ & + & - & - \\
\hline ST20 ( $\Delta s m c$, Phyper-parE) & $0 \cdot 1$ & 16 & + & - & - \\
\hline ST20 ( $\Delta s m c$, Phyper-parE) & 1 & $5 \cdot 5$ & + & + & - \\
\hline
\end{tabular}

${ }^{\star}$ Until cell growth arrested.

\section{Deletions of $s m c, \operatorname{scp} A$ or $\operatorname{scp} B$ differentially affect global protein synthesis}

The link between Topo IV and the SMC protein prompted us to investigate if the SMC complex has a global effect on supercoiling, that is, if it influences global transcription throughout the chromosome, in a similar manner to Topo I, Topo IV and DNA gyrase. We chose an indirect approach, through analysing the pattern of protein expression, which is tightly linked to rates of transcription in bacteria. We performed 2D gel analysis of cells growing exponentially at $23^{\circ} \mathrm{C}$ in rich medium (which is permissive for $s m c, s c p A$ and $s c p B$ mutant cells), and analysed the rate of synthesis of cytosolic proteins. We were able to detect 420 discrete protein spots, corresponding to approximately the same number of proteins, in cell extracts from wild-type and mutant cells (Fig. 3). Of these, 81 reproducibly showed different levels of expression (that is in at least two independent experiments), with 46 spots being expressed at a lower level in the $s m c$ mutant cells, and 35 having a higher expression level than that of wild-type cells (Fig. 3A, B). Thus, the synthesis of at least $20 \%$ of the cytosolic proteins was affected by the $s m c$ deletion. Likewise, 82 protein spots had considerably altered levels in the $\operatorname{sp} B$ mutant strains (Fig. 3C), as well as a similar number in scpA mutant cells (data not shown). Strikingly, many proteins that were down-regulated in $s m c$ mutant cells had a higher level in $s c p B$ mutant cells, compared to wild-type cells, and vice versa. For example, EF-G had a lower level in smc mutant cells, but showed an increased level in $s c p B$ mutant cells, while the opposite was true for spot 11 (Table 2). Thus, loss of different parts of the SMC complex had an effect on the synthesis of different groups of proteins.

Based on the previously established 2D gel index for $B$. subtilis (Eymann et al., 2004; Graumann et al., 1996), it was possible to identify several of the proteins with an altered expression level. Only a representative number of protein spots are marked in Fig. 3. The spots could be roughly grouped into four categories: firstly, proteins downregulated in $s m c$ and in $s c p B$ mutant cells (most notably, the GroEL chaperone and translation factors/proteins EF-G, L10 and S1, although EF-G is upregulated in $s c p B$ mutant cells, Table 2); secondly, proteins with lower levels in $s c p B$ mutant cells than those of wild-type cells, but with similar expression levels between wild-type cells and $s m c$ mutant cells (representatives are SOD, EF-Tu, PdcH and spots 12 13 , Table 2); thirdly, spots with a higher level in $s c p B$ mutant cells that were unaltered in $s m c$ mutant cells (representatives are spots 5-8); and fourthly, spots with higher expression in $s m c$ mutant cells (compared to wild-type), but unaltered levels in $s c p B$ mutant cells (e.g. spots 1, 3, 4, 9-11). Thus, although there is a considerable overlap of proteins with altered expression in $s m c$ and $s c p B$ mutant cells, there are notable differences.

\section{Increased synthesis of Topo IV rescues the defect in protein synthesis in smc mutant cells}

The link between SMC protein and Topo IV with respect to chromosome compaction prompted us to investigate if an increase in Topo IV level in smc mutant cells might influence global protein synthesis via an effect on transcription. Strikingly, at $1 \mathrm{mM}$ IPTG, phyperspank-parE smc mutant cells showed a pattern of protein synthesis that closely resembles that of wild-type cells (Fig. 3D). Of the 81 protein spots that showed increased or reduced intensity in $s m c$ mutant cells compared to wild-type cells, upon the overexpression of Topo IV, 74 had an intensity closely resembling that of wild-type cells. Except for S6, which had a lower expression in smc null and $s c p B$-deleted cells, all of the marked spots in Fig. 3 had a similar intensity between wildtype and $s m c$ null cells with overexpressed Topo IV. To verify our visual observations, we employed the Proteomweaver 2.1 software, which compares and evaluates spot intensities from $2 \mathrm{D}$ gels. Table 3 shows that there was a highly significant difference $(P<0.05)$ in overall protein synthesis between wild-type (PY79) and smc mutant (P 4388 ) cells for a large number of the spots analysed. Likewise, $s m c$ mutant cells and ST20 cells ( $s m c$ null cells in which Topo IV is overproduced) showed a highly significant difference in global expression levels, while there was no 

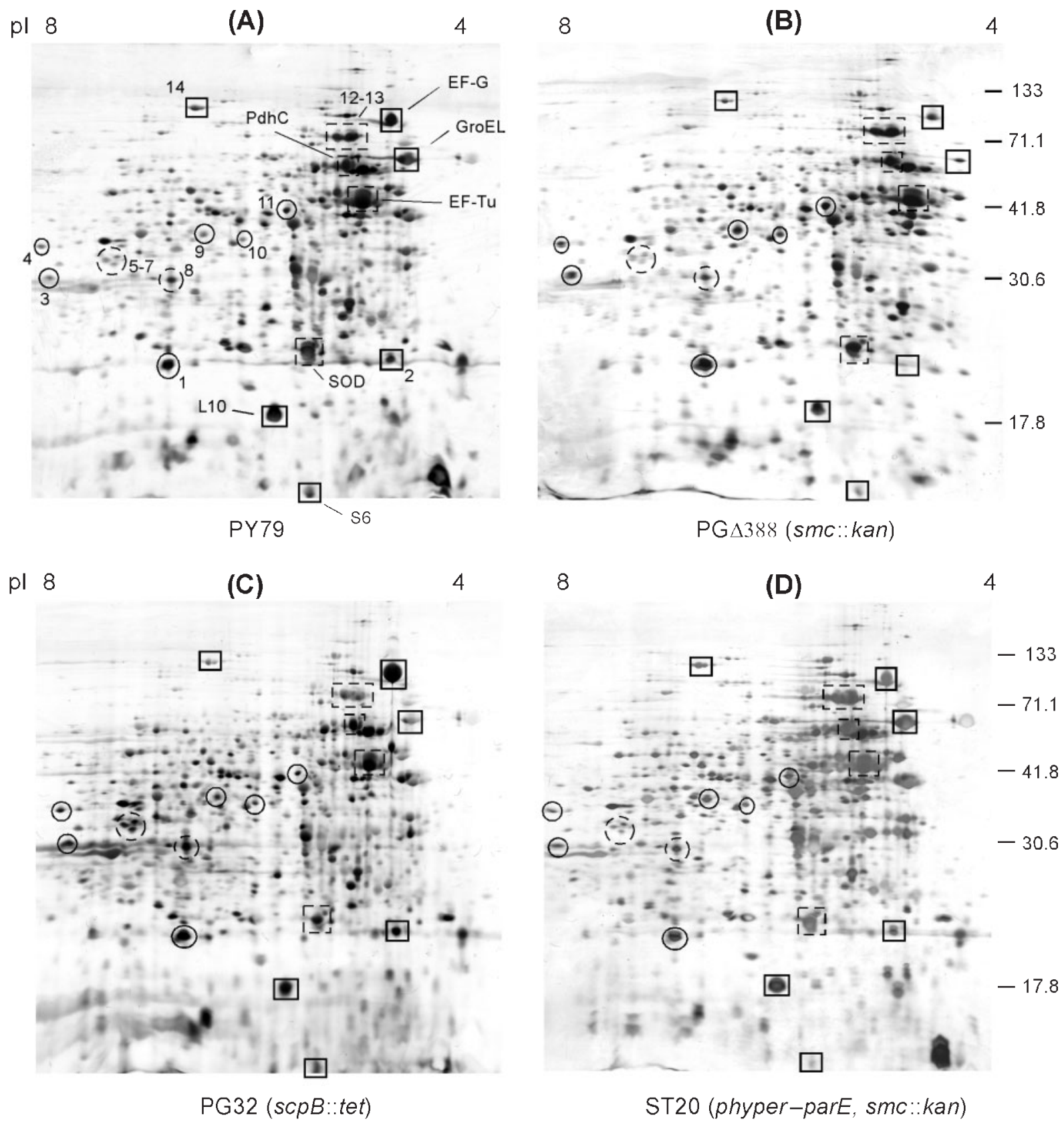

Fig. 3. Analysis of global protein synthesis in wild-type and different mutant strains at $23^{\circ} \mathrm{C}$. (A) Wild-type, (B) smc deletion strain, (C) $s c p B$ deletion strain, (D) smc deletion strain in which Topo IV is overproduced (1 mM IPTG). Solid squares indicate proteins that are expressed at lower levels in smc mutant cells compared to wild-type cells; solid circles show examples of proteins expressed at higher levels in smc mutant cells compared to wild-type cells; dashed squares and circles show proteins that are expressed at higher or lower levels in $s c p B$ mutant cells compared to smc mutant cells (as well as to wildtype cells). Numbers on the right of the gels indicate protein markers; the $\mathrm{pH}$ range is shown at the top of the gels.

significant overall difference between PY79 and ST20 cells (Table 3, $P>0 \cdot 05$ ). However, statistically, there was no major difference between $s m c$ and $\operatorname{scp} B$ (PG32) mutant cells (Table 3, $P>0.05$ ), showing that although several proteins showed different expression levels between the mutant strains, most of the proteins had similar levels (i.e. similarly different levels from wild-type cells). These data analyses verify the conclusions drawn from the visual inspection of the $2 \mathrm{D}$ gels and show that increased production of Topo IV in $s m c$ mutant cells results in protein expression that is similar to that in wild-type cells and thus rescues the defect in protein synthesis in $s m c$ mutant cells. Because Topo IV 
Table 2. Proteins whose synthesis is influenced in different genetic backgrounds

$>$, Higher level; <, lower level; , comparable level.

\begin{tabular}{|c|c|c|c|}
\hline \multirow[t]{2}{*}{ Protein or spot no. } & \multicolumn{3}{|c|}{$\begin{array}{l}\text { Level relative to } \\
\text { wild-type for mutant: }\end{array}$} \\
\hline & $\Delta s m c$ & $\Delta s c p B$ & $\begin{array}{c}\Delta s m c, \\
\text { phyper-parE }\end{array}$ \\
\hline S6 & $<$ & $\sim$ & $<$ \\
\hline L10 & $<$ & $<$ & $\sim$ \\
\hline 2 & $<$ & $\sim$ & $\sim$ \\
\hline GroEL & $<$ & $<$ & $\sim$ \\
\hline EG-G & $<$ & $>$ & $\sim$ \\
\hline 14 & $<$ & $<$ & $\sim$ \\
\hline $\begin{array}{l}\text { SOD (superoxide } \\
\text { dismutase) }\end{array}$ & $\sim$ & $<$ & $\sim$ \\
\hline $\mathrm{EF}-\mathrm{Tu}$ & $\sim$ & $<$ & $\sim$ \\
\hline PdhC & $\sim$ & $<$ & $\sim$ \\
\hline $12-13$ & $\sim$ & $<$ & $\sim$ \\
\hline $5-7$ & $\sim$ & $>$ & $\sim$ \\
\hline 8 & $\sim$ & $>$ & $\sim$ \\
\hline 1 & $>$ & $\sim$ & $\sim$ \\
\hline 3 & $>$ & $>$ & $\sim$ \\
\hline 4 & $>$ & $\sim$ & $\sim$ \\
\hline 9 & $>$ & $\sim$ & $\sim$ \\
\hline 10 & $>$ & $\sim$ & $\sim$ \\
\hline 11 & $>$ & $\sim$ & $\sim$ \\
\hline
\end{tabular}

acts at the level of transcription, these data suggest that the defect in protein synthesis in $s m c$ mutant cells is based on a defect in transcription, due to loss of supercoiling, which is compensated by an increase in Topo IV activity. Moreover, these data show that Topo IV can rescue cell growth in $s m c$ mutant cells largely by suppressing the defect in supercoiling (and thus the defect in transcription), but cannot fully rescue the growth defect, since the defect in chromosome segregation is still severe.

\section{DISCUSSION}

This work establishes the existence of a genetic link between B. subtilis Topo IV and the SMC complex, and provides several important conclusions. Firstly, overproduction of Topo IV leads to hypercompaction of the whole B. subtilis chromosome, whereas decreased levels induce chromosome decondensation. Thus, it is clear that Topo IV acts throughout the nucleoid, and not only at specific sites, which is thought to be the case for E. coli Topo IV (Espeli et al., 2003). These data are consistent with our recent finding that both subunits of Topo IV localize throughout the nucleoids, without any apparent concentration at distinct sites (S. Tadesse and P. L. Graumann, unpublished results). Secondly, we found that increased synthesis of Topo IV restores chromosome compaction in $s m c$ null cells. Because the loss of SMC protein leads to an increase in positive supercoiling in vivo (Sawitzke \& Austin, 2000), our results suggest that the action of Topo IV increases overall negative supercoiling in vivo, most likely by removing positive supercoils. Additionally, and most importantly, our work establishes that the SMC complex interacts with Topo IV at a genetic level, suggesting that global supercoiling and chromosome compaction is achieved by the combined effects of Topo I, DNA gyrase, Topo IV and the SMC complex. Thirdly, our results establish that the SMC complex has a profound influence on global protein synthesis, most likely via its effect on supercoiling. The pattern of protein synthesis was strikingly different between wild-type and $s m c, s c p A$ and $s c p B$ mutant cells: at least $20 \%$ of cytosolic proteins were synthesized at considerably different levels in the mutant cells. Interestingly, loss of ScpA or ScpB had overlapping and yet somewhat distinct effects on protein synthesis, compared to loss of SMC protein, indicating that the components of the SMC complex confer distinct functions. It remains to be investigated if the different effects on transcription are due to a differential effect on supercoiling [the deletion of $s m c$ is more severe than that of $\operatorname{scp} A$ or $\operatorname{scp} B$ (Mascarenhas et al., 2002)], or if there are more specific contributions of ScpA and ScpB. Although the isolated Scp proteins do not show any DNA

Table 3. Statistical comparison of spots of different strains from the 2D gel assay

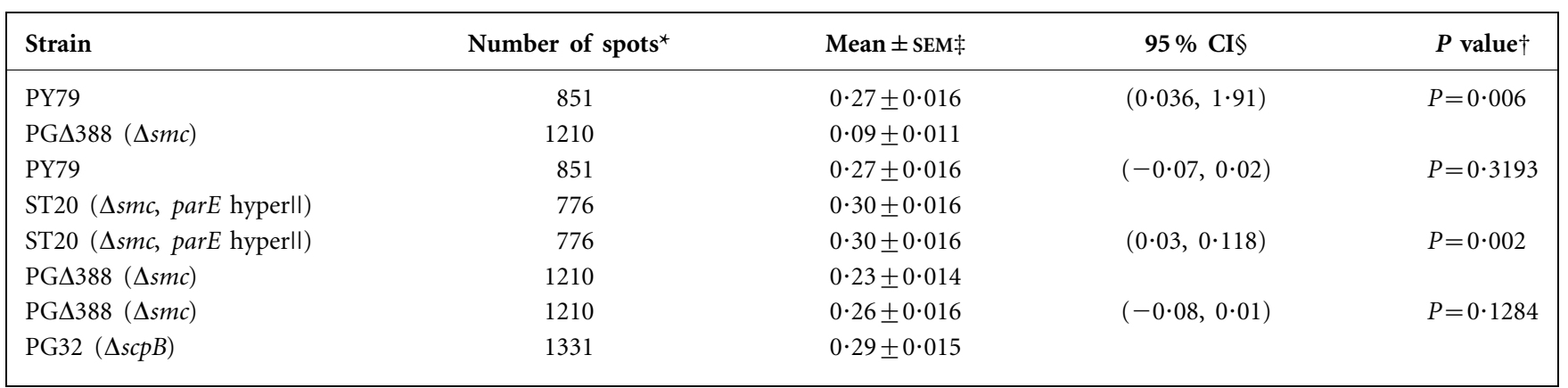

${ }^{\star}$ Analysed; $\dagger P<0.05$ statistically significant, $P>0.05$ not significant; $\ddagger$ standard error of the mean; \$confidence interval; $I 11 \mathrm{mM}$ IPTG, i.e. overproduction of Topo IV. 
binding activity, ScpB contains a helix-turn-helix motif, and could be involved in transcription regulation via additional proteins. Interestingly, ScpA has been shown to interact with transcription factors and several additional proteins in a two-hybrid system, and to affect the function of a two-component system regulating sugar metabolism (Dervyn et al., 2004). In any event, the defect in protein synthesis in $s m c$ mutant cells could be almost entirely cured by overproduction of Topo IV, indicating that the defect of the $s m c$ deletion is due to a lack of supercoiling, which can be rescued by increased Topo IV activity. Thus, SMC proteins have a genome-wide effect on protein synthesis in a prokaryote, which extends the action of SMC proteins, in addition to their role in the transcriptional silencing of a whole chromosome in Caenorhabditis elegans (Chuang et al., 1994).

Fourthly, our work suggests that the SMC complex has a dual function: in chromosome compaction and protein synthesis (via general supercoiling), which can be suppressed by overproduction of Topo IV; and in active chromosome segregation, which can only partially be rescued by Topo IV, because the formation of anucleate cells is reduced by only half through overproduction of Topo IV. Even if general supercoiling is brought to wild-type levels, chromosomes still fail to be separated in $s m c$ mutant cells, accompanied by slower growth and temperature sensitivity above $30^{\circ} \mathrm{C}$ (compared to wild-type cells). Possibly, the special function of the SMC complex in chromosome segregation is brought about by the ability to form discrete subcellular centres on the nucleoids, predominantly one in each cell half (Mascarenhas et al., 2002), from which the complex influences compaction of the whole chromosome (Volkov et al., 2003), and also mediates its crucial function in the segregation of chromosomes after the separation of origin regions (Graumann, 2000). We favour the view that from the middle of the cell (where replication takes place), newly replicated DNA is somehow brought to the bipolar SMC condensation centres, where DNA from each sister chromosome is locally condensed, and thereby pulled into each cell half. We have recently found evidence that in vitro SMC protein forms rosette-like structures containing multiple SMC protein molecules (Mascarenhas et al., 2005), which may connect many DNA loops bound by individual SMC protein dimers, and which could be the basis for the formation of the observed condensation centres in vivo.

In toto, our results suggest that the bacterial nucleoid is compacted through a combined action of Topo I, SMC complex, DNA gyrase and Topo IV, and that the latter three introduce net negative supercoiling, which is counterbalanced by Topo I. The genetic interactions with DNA gyrase and Topo IV underline the intricate interplay of the SMC complex with topoisomerases, yet reveal the distinct role of the SMC complex in active chromosome segregation. The molecular bases underlying these processes will be interesting to study in detail in future work.

\section{ACKNOWLEDGEMENTS}

This work was supported by the Deutsche Forschungsgemeinschaft (Emmy Noether and Heisenberg Programm) and by BMBF grant 031U204B.

\section{REFERENCES}

Chuang, P. T., Albertson, D. G. \& Meyer, B. J. (1994). DPY-27: a chromosome condensation protein homolog that regulates C. elegans dosage compensation through association with the $\mathrm{X}$ chromosome. Cell 79, 459-474.

Dervyn, E., Noirot-Gros, M. F., Mervelet, P., McGovern, S., Ehrlich, S. D., Polard, P. \& Noirot, P. (2004). The bacterial condensin/ cohesin-like protein complex acts in DNA repair and regulation of gene expression. Mol Microbiol 51, 1629-1640.

Espeli, O. \& Marians, K. J. (2004). Untangling intracellular DNA topology. Mol Microbiol 52, 925-931.

Espeli, O., Levine, C., Hassing, H. \& Marians, K. J. (2003). Temporal regulation of topoisomerase IV activity in E. coli. Mol Cell 11, 189-201.

Eymann, C., Dreisbach, A., Albrecht, D. \& 10 other authors (2004). A comprehensive proteome map of growing Bacillus subtilis cells. Proteomics 4, 2849-2876.

Graumann, P. L. (2000). Bacillus subtilis SMC is required for proper arrangement of the chromosome and for efficient segregation of replication termini but not for bipolar movement of newly duplicated origin regions. J Bacteriol 182, 6463-6471.

Graumann, P., Schroder, K., Schmid, R. \& Marahiel, M. A. (1996). Cold shock stress-induced proteins in Bacillus subtilis. J Bacteriol 178, 4611-4619.

Gruber, S., Haering, C. H. \& Nasmyth, K. (2003). Chromosomal cohesin forms a ring. Cell 112, 765-777.

Hirano, T. (2002). The ABCs of SMC proteins: two-armed ATPases for chromosome condensation, cohesion, and repair. Genes Dev 16, 399-414.

Jaacks, K. J., Healy, J., Losick, R. \& Grossman, A. D. (1989). Identification and characterization of genes controlled by the sporulation regulatory gene spoOH in Bacillus subtilis. J Bacteriol 171, 4121-4129.

Kato, J., Nishimura, Y., Imamura, R., Niki, H., Hiraga, S. \& Suzuki, H. (1990). New topoisomerase essential for chromosome segregation in E. coli. Cell 63, 393-404.

Kimura, K., Rybenkov, V. V., Crisona, N. J., Hirano, T. \& Cozzarelli, N. R. (1999). $13 \mathrm{~S}$ condensin actively reconfigures DNA by introducing global positive writhe: implications for chromosome condensation. Cell 98, 239-248.

Klein, J., Harding, G. \& Klein, E. (2002). A new isoelectric focusing gel for two-dimensional electrophoresis constructed in microporous hollow fiber membranes. J Proteome Res 1, 41-45.

Lemon, K. P. \& Grossman, A. D. (2000). Movement of replicating DNA through a stationary replisome. Mol Cell 6, 1321-1330.

Lindow, J. C., Britton, R. A. \& Grossman, A. D. (2002a). Structural maintenance of chromosomes protein of Bacillus subtilis affects supercoiling in vivo. J Bacteriol 184, 5317-5322.

Lindow, J. C., Kuwano, M., Moriya, S. \& Grossman, A. D. (2002b). Subcellular localization of the Bacillus subtilis structural maintenance of chromosomes (SMC) protein. Mol Microbiol 46, 997-1009.

Mascarenhas, J., Soppa, J., Strunnikov, A. \& Grauman, P. L. (2002). Cell cycle dependent localization of two novel prokaryotic 
chromosome segregation and condensation proteins in Bacillus subtilis that interact with SMC protein. EMBO J 21, 3108-3118.

Mascarenhas, J., Volkov, A. V., Rinn, C., Schiener, J., Guckenberger, R. \& Graumann, P. L. (2005). Dynamic assembly, localization and proteolysis of the Bacillus subtilis SMC complex. BMC Cell Biology 6, 28 (http://www.biomedcentral.com/1471-2121/6/28).

Quisel, J. D., Burkholder, W. F. \& Grossman, A. D. (2001). In vivo effects of sporulation kinases on mutant Spo0A proteins in Bacillus subtilis. J Bacteriol 183, 6573-6578.

Sawitzke, J. A. \& Austin, S. (2000). Suppression of chromosome segregation defects of Escherichia coli muk mutants by mutations in topoisomerase I. Proc Natl Acad Sci U S A 97, 1671-1676.

Soppa, J., Kobayashi, K., Noirot-Gros, M. F., Oesterhelt, D., Ehrlich, S. D., Dervyn, E., Ogasawara, N. \& Moriya, S. (2002). Discovery of two novel families of proteins that are proposed to interact with prokaryotic SMC proteins, and characterization of the Bacillus subtilis family members ScpA and ScpB. Mol Microbiol 45, 59-71.

Volkov, A., Mascarenhas, J., Andrei-Selmer, C., Ulrich, H. D. \& Graumann, P. L. (2003). A prokaryotic condensin/cohesin-like complex can actively compact chromosomes from a single position on the nucleoid and binds to DNA as a ring-like structure. Mol Cell Biol 23, 5638-5650.

Zechiedrich, E. L., Khodursky, A. B., Bachellier, S., Schneider, R., Chen, D., Lilley, D. M. \& Cozzarelli, N. R. (2000). Roles of topoisomerases in maintaining steady-state DNA supercoiling in Escherichia coli. J Biol Chem 275, 8103-8113.

Zhu, Q., Pongpech, P. \& DiGate, R. J. (2001). Type I topoisomerase activity is required for proper chromosomal segregation in Escherichia coli. Proc Natl Acad Sci U S A 98, 9766-9771. 